\title{
Is surgery after neoadjuvant chemoradiotherapy feasible for elderly patients with resectable or borderline resectable pancreatic ductal adenocarcinoma?
}

Hironobu Suto ( $\square$ hsuto@med.kagawa-u.ac.jp )

Kagawa University https://orcid.org/0000-0001-8476-3985

Keiichi Okano

Kagawa University

Minoru Oshima

Kagawa University

Yasuhisa Ando

Kagawa University

Hiroyuki Matsukawa

Kagawa University

Shigeo Takahashi

Kagawa University

Toru Shibata

Kagawa University

Hideki Kamada

Kagawa University

Hideki Kobara

Kagawa University

Tsutomu Masaki

Kagawa University

Yasuyuki Suzuki

Kagawa University

\section{Research article}

Keywords: elderly patients, neoadjuvant chemoradiotherapy, adjuvant chemotherapy, pancreatic ductal adenocarcinoma

Posted Date: January 17th, 2020 
DOI: https://doi.org/10.21203/rs.2.21112/v1

License: (c) (1) This work is licensed under a Creative Commons Attribution 4.0 International License. Read Full License 


\section{Abstract}

\section{Background}

The benefit and safety of pancreas resection for pancreatic ductal adenocarcinoma for elderly patients, especially after preoperative adjuvant therapy, is still unknown. This study attempted to evaluate perioperative and long-term outcomes after pancreas resection in elderly patients with pancreatic ductal adenocarcinoma and to detect the potential impact of neoadjuvant chemoradiotherapy.

\section{Methods}

One hundred and thirty-four consecutive patients undergoing curative resection for resectable and borderline resectable pancreatic ductal adenocarcinoma between March 2008 and February 2018 at our institution were analyzed. Patients were divided into two groups: patients older than or equal to 75 years (the elderly group, $n=46$ ) and those younger than 75 years (the younger group, $n=88$ ).

\section{Results}

There were no significant differences both in overall survival and relapse free survival between the two groups $(P=0.270, P=0.699)$. Although the induction rate of adjuvant chemotherapy was not significantly different $(P=0.458)$, the completion rate was significantly lower in elderly group than that in younger group (35\% and $56 \% ; P=0.022)$. Neoadjuvant chemoradiotherapy was performed for 82 patients $(61 \%)$, and the induction and completion rates were not significantly different $(P=0.668, P=0.794)$ between the two groups. The elderly patients with completion of adjuvant chemotherapy had significantly better overall survival than those without it $(P=0.032)$. Neoadjuvant chemoradiotherapy did not significantly affect overall survival in elderly patients, however, there was a trend toward longer overall survival in patients who had neoadjuvant chemoradiotherapy $(P=0.072)$.

\section{Conclusions}

Neoadjuvant chemoradiotherapy could be introduced and completed even for elderly patients without serious complications and might lead to improved prognosis for those who are difficult to complete postoperative adjuvant chemotherapy.

\section{Background}

With advances in perioperative management and surgical techniques in recent years, pancreas resection has become accepted as a safe and effective procedure even in elderly patients with acceptable morbidity and mortality rates. Several reports have emphasized that pancreaticoduodenectomy (PD) for elderly patients could be beneficial, as it is in younger patients [1-3]. However, large population-based studies showed a mortality of 4.5-15.5\% after pancreas resection in patients aged 80 years or older [46]. A few recent series from large centers have indicated that pancreas resections should not be avoided for the reason of age itself even in elderly patients in terms of the short-term outcomes [7-11]. On the 
other hand, Ogura et al [6] suggested that PD for pancreatic ductal adenocarcinoma (PDAC) in elderly patients should be carefully selected because it is associated with a higher incidence of severe postoperative complications and a small change of long-term survival. Thus, the efficacy and benefit of pancreas resections for PDAC in elderly patients remain controversial because it has been reported that the elderly patients with PDAC had a limited prognosis even though pancreas resection is the only curative treatment option $[8,9]$.

The CONKO-001 and JASPAC01 studies suggested that postoperative adjuvant chemotherapy (ACT) had an important effect in patients with PDAC $[12,13]$. However, one-fourth of patients with PDAC postoperatively cannot receive ACT because of insufficient recovery or surgery-related complications [14], and this tendency is particularly evident among elderly patients.

Preoperative neoadjuvant therapy was not actually recommended for patients with resectable (R) PDAC in the NCCN guideline [15]. However, we already reported the efficacy and safety of preoperative neoadjuvant chemoradiotherapy (NACRT) in patients with R and borderline resectable (BR) PDAC [16]. Furthermore, our study also showed a high completion rate of NACRT. NACRT might compensate for a lack of such a postoperative adjuvant chemotherapy, especially for elderly patients with difficulty in induction and completion of ACT.

The aim of this study was to evaluate a safety and indication of pancreas resections in elderly patients in comparison with those in younger patients and to detect the influence of preoperative therapy, especially focusing on NACRT.

\section{Methods}

This study was retrospectively analyzed. The Institutional Review Board of Kagawa University approved the study. A total of 150 consecutive patients undergoing pancreas resections for PDAC between March 2008 and February 2018 were retrospectively examined. All 150 patients had PDAC that was histologically confirmed by at least two pathologists.

\section{Patients}

Of the 150 patients, 16 patients were excluded. Fourteen were classified as unresectable category based on the NCCN guideline [15], one underwent R2 resection, and one underwent preoperative therapy other than NACRT. The data from the remaining 134 patients were retrospectively analyzed.

The patients were diagnosed with R-PDAC $(n=114)$ or BR-PDAC $(n=20)$. All surgical procedures were divided into the following three types: classic, pylorus-preserving, or subtotal stomach-preserving pancreaticoduodenectomy (PD) in 90 patients (67\%); distal pancreatectomy in 37 (28\%); and total pancreatectomy in 7 (5\%). Patients who underwent laparoscopic surgery were not included in this series. Systematic lymph node dissection was performed in all operations. R0 resection was achieved in 128 
patients $(96 \%)$ and R1 was achieved in $6(4 \%)$. Preoperative NACRT and postoperative ACT were given to $82(61 \%)$ and $104(78 \%)$ patients, respectively.

Patients were divided into two groups: patients older than or equal to 75 years (the elderly group, $n=46$ ) and those younger than 75 years (the younger group, $n=88$ ).

\section{Preoperative NACRT}

We introduced two kinds of NACRT during the period of this study. Short-term neoadjuvant hypofractionated chemoradiotherapy with S1 was performed between January 2009 and May 2016 as a prospective phase II trial, and already reported its efficacy and safety [16]. In this clinical trial, hypofractionated, external-beam radiotherapy (30 Gy in 10 fractions) with concurrent $\mathrm{S} 1\left(60 \mathrm{mg} / \mathrm{m}^{2}\right)$ was delivered 5 days per week for 2 weeks prior to pancreatectomy. Since June 2016, the next phase II trial is underway with external-beam radiotherapy (50 Gy in 25 fractions) and concurrent $\mathrm{S} 1\left(60 \mathrm{mg} / \mathrm{m}^{2}\right.$ ) for 5 weeks. Short-term and extended NACRT were given to 54 and 28 patients, respectively.

\section{Postoperative ACT and follow-up}

Postoperative ACT was applied postoperatively unless contraindicated by the patients' conditions. Basically, the patients received gemcitabine, referring to the results of the CONKO-001 trial [12] between 2008 and 2012; or S-1, referring to the results of the JASPAC01 trial since 2013 [13], according to the recommended protocols.

The follow-up examinations were performed every 2-3 months for 1 year and every 6 months thereafter. Enhanced computed tomography was performed every 6 months. We moved the examination date forward or added magnetic resonance imaging or ${ }^{18} \mathrm{~F}$-fluorodeoxy glucose positron emission tomography (FDG-PET), if necessary.

\section{Outcome measures}

The preoperative variables included age; sex; body mass index (BMI); tumor location; resectability [15]; serum C-reactive protein (CRP), serum albumin, hemoglobin, and serum CA19-9 levels; neutrophil / lymphocyte ratio (NLR); lymphocyte count; modified Glasgow Prognostic Score [17]; the standardized uptake value (SUV) seen on FDG-PET; and induction or completion rate of NACRT. The intraoperative data, including the surgical procedure, operation time, estimated blood loss, blood transfusion, and portal vein resection and pathological variables were also collected to compare the elderly and younger groups. Postoperative data on morbidity according to the Clavien-Dindo (CD) classification [18], postoperative pancreatic fistula (POPF) [19], delayed gastric emptying (DGE) [20], and induction and completion rate of postoperative ACT were included.

\section{Statistical analysis}

The clinicopathological features of patients in the elderly and younger groups were compared. The categorical variables were compared between the groups using the chi-square test and Fisher's exact test. 
Survival was calculated using the Kaplan-Meier method and was compared between the groups using the log-rank test. P-values $<0.05$ were considered statistically significant. All statistical analyses were performed using the SPSS Statistics 25.0 for Windows software program (SPSS, Inc., Chicago, IL, USA).

\section{Results}

The median follow-up period was 21 (range: 0-121) months. In the entire group of patients, the median OS was 34 months, and the 3 - and 5-year survival rates were $48.5 \%$ and $36.9 \%$, respectively.

Among the 134 patients undergoing pancreas resections for PDAC, 46 patients (34\%) were aged 75 years or older. The comparison of clinicopathologic characteristics and postoperative outcomes between the elderly and younger patients was shown in Table 1. Although the elderly patients had more risk and trend with aspect to hemoglobin level and NLR $(P=0.010, P=0.062)$, there were no significant differences in the induction and completion rates of NACRT, intraoperative variables including the surgical procedures, incidence of portal vein resection, length of operation and blood loss, and pathological data of LN metastasis and resection status between the two groups.

Mortality occurred in a patient in the younger group. Morbidity [18] ( $\geq$ Grade Illb) occurred in 7 (15\%) in the elderly group. The elderly group experienced a significantly higher incidence of postoperative major complications $(P=0.046)$. The incidence of POPF was similar between the two groups, while DGE occurred more frequently in the elderly patients than in the younger patients $(P=0.001)$. Furthermore, postoperative hospital stay in

the elderly group was longer than that in the younger group $(P=0.008)$. The induction rate of ACT was similar between the two groups ( $74 \%$ vs $80 \%, P=0.458)$, however, the completion (more than 6 months) rate was significantly lower in the elderly group than the younger group $(P=0.022)$. Only $16(35 \%)$ patients could complete ACT in the elderly group.

NACRT was performed for 82 patients (61\%). Twenty-seven (59\%) patients in the elderly group received NACRT ( 2 weeks: $n=18$ and 5 weeks: $n=9$ ), and $55(63 \%)$ in the younger group did ( 2 weeks: $n=36$ and 5 weeks: $n=19)$. There were no significant differences about the induction of NACRT between the two groups $(P=0.668)$. As for the completion rate, 24 out of $27(89 \%)$ completed NACRT protocol in the elderly group and 48 out of $55(87 \%)$ in the younger group. There were no significant differences about the completion rate of NACRT between the two groups. More importantly, both groups had high completion rate of NACRT regardless of the treatment period.

The median overall survival (OS) time and 3-year OS rate in the elderly patients were 27 months and $45 \%$, compared to 58 months and $52 \%$ in the younger patients, respectively (Fig. 1a). As well, the median relapse free survival time and 3-year relapse free survival rate (RFS) in the elderly patients were 18 months and $36 \%$, compared to 16 months and $36 \%$ in the younger patients, respectively (Fig. 1b). The differences were not statistically significant both in OS and RFS between the two groups $(P=0.270, P=$ 0.699). 
Table 2 shows the subgroup analysis for the clinicopathological factors in elderly patients with $(n=27)$ and without $(n=19)$ NACRT. The background factors such as age, sex, BMI, resectability [15], serum albumin and hemoglobin, NLR, intraoperative blood loss, transfusion, pathological LN metastases and resection status were not significantly different between the patients with and without NACRT. PD and TP were more frequently performed in patients with NACRT $(P=0.014)$. Portal vein was more frequently resected in the NACRT group $(P=0.044)$, and operation time was significantly longer in patients with NACRT $(P=0.049)$. As for perioperative outcomes, there were no significantly differences in morbidity, postoperative hospital stay and ACT induction and completion rates between the patients with and without NACRT.

The elderly patients with completion of postoperative ACT had significantly better OS than those without completion or induction of ACT ( $P=0.032$ ) (Fig. 2). The median OS and 3-year OS rate in the elderly patients with completion of ACT were 45 months and $66 \%$, compared to 18 months and $33 \%$ in its counterpart. On the other hand, NACRT itself did not significantly affect OS in elderly patients, however, there was a trend toward improvement of OS $(P=0.072)$ (Fig. 3).

Figure 4 showed OS curves in the elderly group dividing into 4 groups with and without inductions of NACRT and ACT. Only patients who introduced both NACRT and ACT had significantly better OS than those who did either of NACRT or ACT or did not do either $(P=0.042, P=0.017$ and $P=0.002)$. It was suggested that performing NACRT before surgery and further performing ACT after surgery would improve OS for the elderly patients.

\section{Discussion}

Some studies have reported that even elderly patients achieved comparable short-term outcomes and similar rates of perioperative complications following pancreatic resections as younger patients thanks to recent improvements in surgical techniques and perioperative care $[10,11]$. And yet at the same time, others demonstrated that a higher incidence of postoperative complication was seen in patients older than 75 or 80 years of age $[5,7-9,21]$. The current study revealed that the frequencies of major morbidity and DGE were significantly higher, and consequently, postoperative hospital stay was longer in the elderly patients. This might suggest that small problems for younger patients easily lead to big problems for elderly patients. Ballarin et al [10] described that age itself was not directly related to morbidity rate but that comorbidities might have a connection with it. They said that the presence of comorbidities such as hyperlipoproteinemia, diabetes, and coronary artery disease might be potential risk factors of morbidity. However, in this series, pancreatic resection was almost safely performed even in the elderly because mortality rate was zero. The careful management and patients' selection in consideration of their backgrounds might be essential.

The efficacy of postoperative ACT was widely known and scientifically proven [12, 13]. However, surgical burden might preclude the induction or completion of ACT, especially for elderly patients. Khan et al [9] asserted that PD for PDAC in elderly patients could not be recommended because additional 
chemoradiation or systemic chemotherapy was often not feasible in the population group. The current study also indicated that the ACT completion rate was significantly lower in the elderly patients than that in the younger patients although the induction rate was similar between the groups.

Recently, the efficacy of preoperative neoadjuvant chemotherapy or CRT for PDAC has been reported, especially for patients with BR- or locally advanced PDAC [22, 23]. Preoperative neoadjuvant therapy is not fully recommended for patients with R-PDAC in the NCCN guideline [15], presumably because of insufficient evidence. However, preoperative therapy possibly has a beneficial effect on patient survival in those with R-PDAC depending on patients. Several previous series [24, 25] and our clinical trial [16] demonstrated that NACRT had survival benefit for patients with R-PDAC as well as BR-PDAC. Additionally, in the current study, NACRT showed a trend toward improvement of OS in the analysis of the elderly group. In contrast to ACT, the completion rate of NACRT was very high even in the elderly group and comparable to the younger counterpart. Furthermore, in the subgroup analyses of the elderly patients, NACRT did not adversely affect perioperative outcomes, and more importantly, NACRT had little influence on induction and completion rate of postoperative ACT.

In this series, NACRT might have contributed to the comparable OS and RFS between the elderly and younger patients with R-and BR-PDAC. NACRT could benefit the elderly patients who had difficulty in the completion of ACT. In our subgroup analysis of OS, however, it was clearly demonstrated that NACRT alone was insufficient and NACRT plus ACT induction could prolong the survival in the elderly patients even if ACT was not completed. From these results, NACRT could be recommended to the elderly patients with PDAC.

We introduced two kinds of NACRT, and it might cause one of the serious limitations in the current study. However, a report from the M.D. Anderson Cancer Center contended that hypofractionated CRT (30 Gy) was associated with margin-negative resection rates, treatment effects, local control, and OS, similar to those associated with standard fractionated CRT (50.4 Gy) [26]. Moreover, there were no differences about the indication and completion rate of the two ways of NACRT between the elderly and younger group, therefore, we regarded them as a NACRT group in the current study.

The current study has several limitations. This was a retrospective study that was conducted at a single institution. Therefore, the sample size was small and a historical backdrop existed, and the current smallsized retrospective study cannot provide enough evidence to draw a definitive conclusion. We defined elderly patients as patients over 75 years old in the current study. However, we did not argue about very elderly patients aged 80 years or older in this study because the sample size of elderly group would become further small. As already mentioned, the two ways of NACRT existed. Ideally, the methods of NACRT should be integrated and analyzed.

\section{Conclusion}

Pancreas resections for elderly patients with PDAC could be safely performed though major complications and DGE rate was higher and the period of postoperative hospital stay became longer, 
compared to younger patients. NACRT could be introduced and completed even for elderly patients without serious problem and might lead to improved prognosis for those who are difficult to complete postoperative adjuvant therapy.

\section{Declarations}

\section{Ethics approval and consent to participate}

All procedures performed in studies involving human participants were in accordance with the ethical standards of the institutional and/or national research committee and with the 1964 Declaration of Helsinki and its later amendments or comparable ethical standards. This study was approved by the institutional review board of Kagawa University, and written informed consent was obtained from all individual participants before surgery for collection and analysis of the data. This article does not contain any studies with animals performed by any of the authors.

\section{Consent for publish}

Not applicable.

\section{Availability of data and materials}

The datasets used and/or analysed during the present study are available from the corresponding author (HS) on reasonable request.

\section{Competing interests}

The authors declare that they have no conflict of interest.

\section{Funding}

This research did not receive any specific grant funding from agencies in the public, commercial, or notfor-profit sectors.

\section{Authors' contributions}

HS operated surgery and performed postoperative management of the patient and wrote the manuscript. MO, YA, HM gathered operated on the patients, gave advice on surgery and revised the manuscript for intellectual content. ST, TS, HK1(Hideki Kamada), HK2(Hideki Kobara) and TM gave substantial contribution to the acquisition, analysis and interpretation of data. KO and YS operated on the patients, and supervised and contributed to the final version of the report. All authors read and approved the final manuscript.

\section{Acknowledgements}


The authors are grateful to all the patients who contributed data to this study. The authors would like to thank Editage (https://www.editage.jp) for the English language review.

\section{Abbreviations}

ACT: adjuvant chemotherapy

Alb: albumin

BMI: body mass index

BR: borderline resectable

CA19-9: carbohydrate antigen 19-9

CD: Clavien-Dindo classification

Cl: Confidence Interval

CRP: C-reactive protein

DGE: delayed gastric emptying

DP: distal pancreatectomy

$\mathrm{Hb}$ : hemoglobin

HR: Hazard Ratio

LN: lymph node

NACRT: neoadjuvant chemoradiotherapy

NLR: neutrophil/lymphocyte ratio

OS: Overall Survival

PD: pancreaticoduodenectomy

PDAC: Pancreatic ductal adenocarcinoma

POPF: postoperative pancreatic fistula

R: resectable

SUV: Standardized Uptake Value 
TP: total pancreatectomy

\section{References}

1. Gouma DJ, van Geenen RC, van Gulik TM, de Haan RJ, de Wit LT, Busch OR, et al. Rates of complications and death after pancreaticoduodenectomy: risk factors and the impact of hospital volume. Ann Surg. 2000;232:786-95.

2. DeOliveira ML, Winter JM, Schafer M, Cunningham SC, Cameron JL, Yeo CJ, et al. Assessment of complications after pancreatic surgery: A novel grading system applied to 633 patients undergoing pancreaticoduodenectomy. Ann Surg. 2006;244:931-7.

3. DiCarlo V, Balzano G, Zerbi A, Villa E. Pancreatic cancer resection in elderly patients. Br J Surg. 1998;85:607-10.

4. Petrowsky H, Clavien PA. Should we deny surgery for malignant hepato-pancreatico-biliary tumors to elderly patients? World J Surg. 2005;29:1093-100.

5. Ballarin R, Spaggiari M, Di Benedetto F, Montalti R, Masetti M, De Ruvo N, et al. Do not deny pancreatic resection to elderly patients. J Gastrointest Surg. 2009;13:341-8.

6. Oguro S, Shimada K, Kishi Y, Nara S, Esaki M, Kosuge T. Perioperative and long-term outcomes after pancreaticoduodenectomy in elderly patients 80 years of age and older. Langenbecks Arch Surg. 2013;398:531-8.

7. Sohn TA, Yeo CJ, Cameron JL, Lillemoe KD, Talamini MA, Hruban RH, et al. Should pancreaticoduodenectomy be performed in octogenarians? J Gastrointest Surg. 1998;2:207-16.

8. Makary MA, Winter JM, Cameron JL, Campbell KA, Chang D, Cunningham SC, et al. Pancreaticoduodenectomy in the very elderly. J Gastrointest Surg. 2006;10:347-56.

9. Khan S, Sclabas G, Lombardo KR, Sarr MG, Nagorney D, Kendrick ML, et al. Pancreatoduodenectomy for ductal adenocarcinoma in the very elderly; is it safe and justified? J Gastrointest Surg. 2010;14:1826-31.

10. Tani M, Kawai M, Hirono S, Ina S, Miyazawa M, Nishioka R, et al. A pancreaticoduodenectomy is acceptable for periampullary tumors in the elderly, even in patients over 80 years of age. $J$ Hepatobiliary Pancreat Surg. 2009;16:675-80.

11. Hatzaras I, Schmidt C, Klemanski D, Muscarella P, Melvin WS, Ellison EC, et al. Pancreatic resection in the octogenarian: a safe option for pancreatic malignancy. J Am Coll Surg. 2011;212:373-7.

12. Oettle H, Neuhaus P, Hochhaus A, Hartmann JT, Gellert K, Ridwelski K, et al. Adjuvant chemotherapy with gemcitabine and long-term outcomes among patients with resected pancreatic cancer: the CONKO-001 randomized trial. JAMA. 2013;310:1473-81.

13. Uesaka K, Boku N, Fukutomi A, Okamura Y, Konishi M, Matsumoto I, et al. Adjuvant Chemotherapy of S-1 Versus Gemcitabine for Resected Pancreatic Cancer: A Phase 3, Open-Label, Randomised, NonInferiority Trial (JASPAC 01). Lancet. 2016;388:248-57. 
14. Heinrich S, Pestalozzi BC, Schafer M, Weber A, Bauerfeind P, Knuth A, et al. Prospective phase II trial of neoadjuvant chemotherapy with gemcitabine and cisplatin for resectable adenocarcinoma of the pancreatic head. J Clin Oncol. 2008;26:2526-31.

15. National Comprehensive Cancer Network Clinical Practice guidelines in oncology pancreatic adenocarcinoma. Available from:

http://www.nccn.org/professionals/physician_gls/pdf/pancreatic.pdf. Accessed 19 December 2019

16. Okano K, Suto H, Oshima M, Maeda E, Yamamoto N, Kakinoki K, et al. A Prospective Phase II Trial of Neoadjuvant S-1 with Concurrent Hypofractionated Radiotherapy in Patients with Resectable and Borderline Resectable Pancreatic Ductal Adenocarcinoma. Ann Surg Oncol. 2017;24:2777-84.

17. La Torre M, Nigri G, Cavallini M, Mercantini P, Ziparo V, Ramacciato G. The Glasgow Prognostic Score as a Predictor of Survival in Patients with Potentially Resectable Pancreatic Adenocarcinoma. Ann Surg Oncol. 2012;19:2917-23.

18. Dindo D, Demartines N, Clavien PA. Classification of Surgical Complications: A New Proposal with Evaluation in a Cohort of 6336 Patients and Results of a Survey. Ann Surg. 2004;240:205-13.

19. Bassi C, Marchegiani G, Dervenis C, Sarr M, Abu Hilal M, Adham M, et al. The 2016 update of the International Study Group (ISGPS) definition and grading of postoperative pancreatic fistula: 11 Years After. Surgery. 2017;161:584-91.

20. Wente MN, Bassi C, Dervenis C, Fingerhut A, Gouma DJ, Izbicki JR, et al. Delayed gastric emptying (DGE) after pancreatic surgery: a suggested definition by the International Study Group of Pancreatic Surgery (ISGPS). Surgery. 2007;142:761-8.

21. Sulpice L, Rayar M, D'Halluin PN, Harnoy Y, Merdrignac A, Bretagne JF, et al. Impact of age over 75 years on outcomes after pancreaticoduodenectomy. J Surg Res. 2012;178:181-7.

22. Katz MH, Pisters PW, Evans DB, Sun CC, Lee JE, Fleming JB, et al. Borderline resectable pancreatic cancer: the importance of this emerging stage of disease. J Am Coll Surg. 2008;206:833-46.

23. Chun YS, Milestone BN, Watson JC, Cohen SJ, Burtness B, Engstrom, et al. Defining venous involvement in borderline resectable pancreatic cancer. Ann Surg Oncol. 2010;17(11):2832-8.

24. Artinyan A, Anaya DA, McKenzie S, Ellenhorn JD, Kim J. Neoadjuvant therapy is associated with improved survival in resectable pancreatic adenocarcinoma. Cancer. 2011;117:2044-9.

25. Papalezova KT, Tyler DS, Blazer DG $3^{\text {rd }}$, Clary BM, Czito BG, Hurwitz HI, et al. Does preoperative therapy optimize outcomes in patients with resectable pancreatic cancer? J Surg Oncol. 2012;106:111-8.

26. Cloyd JM, Crane CH, Koay EJ, Das P, Krishnan S, Prakash L, et al. Impact of hypofractionated and standard fractionated chemoradiation before pancreatoduodenectomy for pancreatic ductal adenocarcinoma. Cancer. 2016;122:2671-9.

\section{Tables}

àdian (min-max) 
Table 1. Comparison of clinicopathologic characteristics and perioperative outcomes between the elderly and younger patients

\begin{tabular}{|c|c|c|c|c|}
\hline & & $\begin{array}{l}\text { Elderly }(\mathrm{n}=46) \\
n \text { (range or } \%)\end{array}$ & $\begin{array}{l}\text { Younger }^{\mathrm{C}}(\mathrm{n}=88) \\
n \text { (range or } \%)\end{array}$ & $P$ value \\
\hline Age (years) & & $79(75-90)^{\mathrm{a}}$ & $67(40-74)^{\mathrm{a}}$ & \\
\hline \multirow[t]{2}{*}{ Sex } & Male & $23(50)$ & $50(57)$ & 0.452 \\
\hline & Female & $23(50)$ & $38(43)$ & \\
\hline BMI $\left(\mathrm{kg} / \mathrm{m}^{2}\right)$ & & $21(15-30)^{a}$ & $22(14-28)^{a}$ & 0.752 \\
\hline \multirow{2}{*}{ Resectability $^{\mathrm{d}}$} & $\mathrm{R}$ & 40 (87) & $74(84)$ & 0.658 \\
\hline & $\mathrm{BR}$ & $6(13)$ & $14(16)$ & \\
\hline Alb & & $3.8(2.5-4.6)^{\mathrm{a}}$ & $3.9(2.8-5.0)^{\mathrm{a}}$ & 0.222 \\
\hline $\mathrm{Hb}$ & & $11.6(8.5-16.3)^{\mathrm{a}}$ & $12.2(8.3-15.7)^{\mathrm{a}}$ & 0.010 \\
\hline NLR & & $3.6(0.8-59.1)^{\mathrm{a}}$ & $2.9(0.9-16.7)^{\mathrm{a}}$ & 0.062 \\
\hline \multirow[t]{3}{*}{ Induction of NACRT } & Yes & $27(59)$ & $55(63)$ & 0.668 \\
\hline & 2 weeks & 18 & 36 & \\
\hline & 5 weeks & 9 & 19 & \\
\hline \multirow[t]{3}{*}{ Completion of NACRT protocol } & Yes & $24(52)$ & $48(55)$ & 0.794 \\
\hline & 2 weeks & 16 & 31 & \\
\hline & 5 weeks & 8 & 17 & \\
\hline \multirow[t]{3}{*}{ Surgical procedure } & $\mathrm{PD}$ & $29(63)$ & $61(69)$ & 0.739 \\
\hline & $\mathrm{DP}$ & $14(30)$ & $23(26)$ & \\
\hline & $\mathrm{TP}$ & $3(7)$ & $4(5)$ & \\
\hline Portal vein resection & & $20(43)$ & $35(39)$ & 0.679 \\
\hline Operation time (min) & & $454(220-710)^{\mathrm{a}}$ & $472(230-816)^{\mathrm{a}}$ & 0.716 \\
\hline Blood loss (ml) & & $1278(152-10564)^{\mathrm{a}}$ & $946(53-9268)^{\mathrm{a}}$ & 0.179 \\
\hline Transfusion & & $18(40)$ & $24(27)$ & 0.160 \\
\hline LN metastases & Positive & $20(43)$ & $41(47)$ & 0.731 \\
\hline Resection status & R0 & $42(91)$ & $86(98)$ & 0.181 \\
\hline Mortality & & $0(0)$ & $1(1)$ & 1.000 \\
\hline Morbidity & & $7(15)$ & $4(5)$ & 0.046 \\
\hline $\operatorname{POPF}^{f}$ (Grade B, C) & & $9(20)$ & $13(15)$ & 0.477 \\
\hline $\mathrm{DGE}^{\mathrm{g}}$ (Grade B, C) & & $20(42)$ & $16(18)$ & 0.001 \\
\hline Postoperative Hospital stay (day) & & $33(14-153)^{\mathrm{a}}$ & $24(10-170)^{\mathrm{a}}$ & 0.008 \\
\hline Induction of ACT & & $34(74)$ & $70(80)$ & 0.458 \\
\hline
\end{tabular}


${ }^{b}$ patients older than or equal to 75 years

${ }^{\mathrm{c}}$ patients younger than 75 years

daccording to the National Comprehensive Cancer Network guideline [15]

${ }^{\text {e}}$ Clavien-Dindo classification [18] $\geq$ grade IIIb

faccording to the International Study Group of Pancreatic Surgery (ISGPS) classification [19]

gaccording to ISGPS classification [20]

aMedian (min-max)

baccording to the National Comprehensive Cancer Network guideline [15]

${ }^{\mathrm{C}}$ Clavien-Dindo classification [18] $\geq$ grade IIIb

daccording to the International Study Group of Pancreatic Surgery (ISGPS) [19]

eaccording to ISGPS classification [20]

\section{Figures}

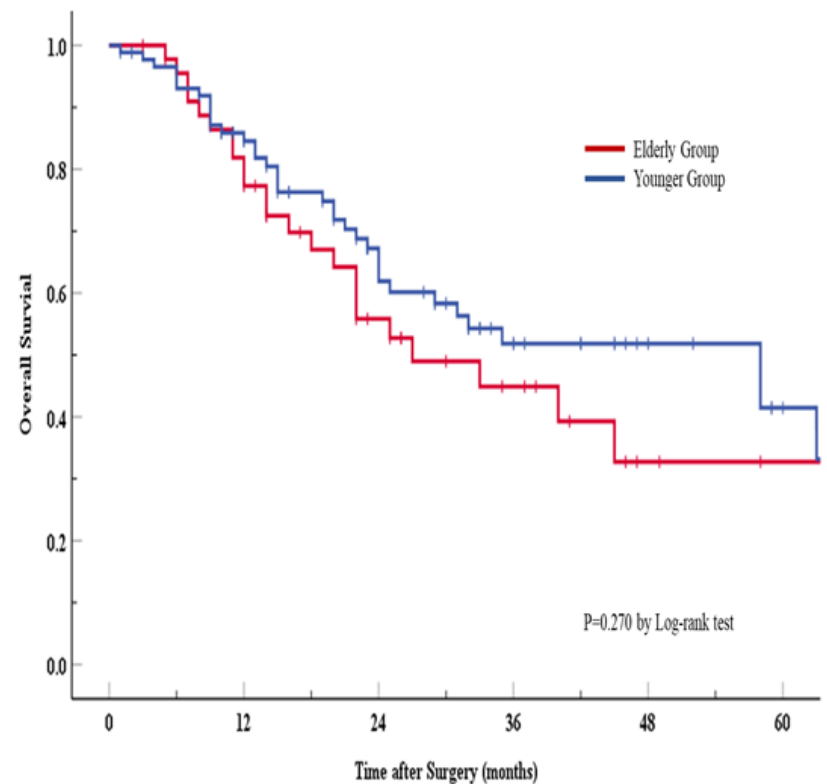

No. at risk

$\begin{array}{lllllll}\text { Elderly Group } & 46 & 35 & 18 & 10 & 3 & 1 \\ \text { Younger Group } & 88 & 64 & 37 & 20 & 11 & 5\end{array}$

a) Comparison of overall survival between the elderly and younger groups

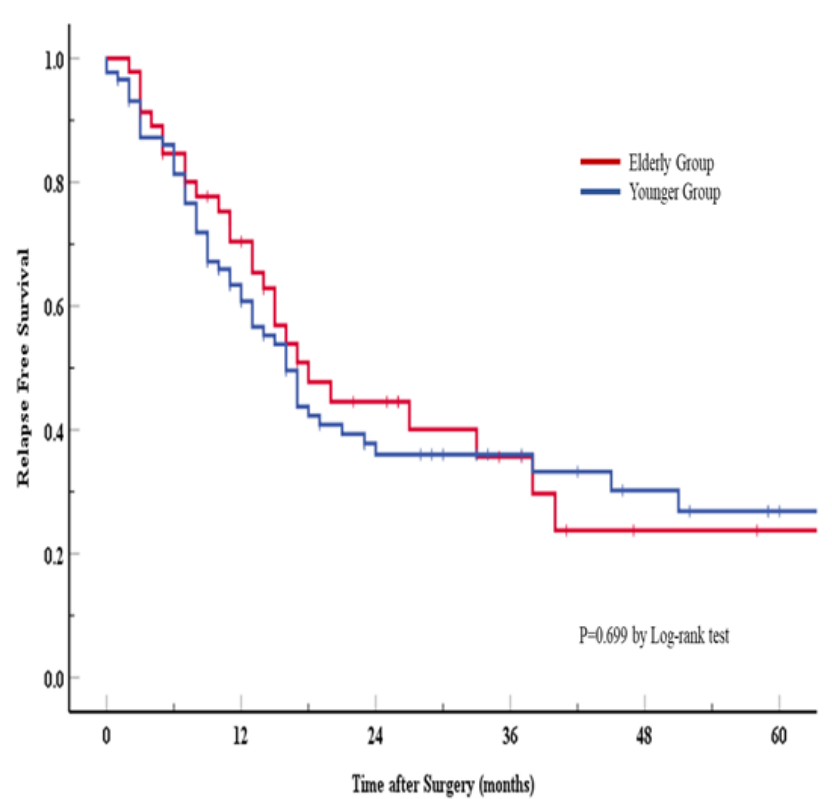

No. at risk

$\begin{array}{lllllll}\text { Elderly Group } & 46 & 34 & 13 & 7 & 2 & 1 \\ \text { Younger Group } & 88 & 47 & 20 & 14 & 9 & 4\end{array}$

b) Comparison of relapse free survival between the elderly and younger groups

Figure 1 
Table 2. Comparison of clinicopathological factors and perioperative outcomes in elderly patients with and without NACRT

\begin{tabular}{|c|c|c|c|c|}
\hline & & $\begin{array}{l}\text { with NACRT }(\mathrm{n}=27 \text { ) } \\
\text { (range or } \% \text { ) }\end{array}$ & $\begin{array}{l}\text { w/o NACRT }(\mathrm{n}=19) \\
\text { (range or } \% \text { ) }\end{array}$ & $P$ value \\
\hline Age (yr) & & $80(75-83)^{a}$ & $78(75-90)^{\mathrm{a}}$ & 0.922 \\
\hline \multirow[t]{2}{*}{ Sex } & Male & $11(41)$ & $12(63)$ & 0.134 \\
\hline & Female & $16(59)$ & $7(37)$ & \\
\hline BMI $\left(\mathrm{kg} / \mathrm{m}^{2}\right)$ & & $22(15-29)^{a}$ & $21(17-30)^{\mathrm{a}}$ & 0.629 \\
\hline \multirow[t]{2}{*}{ Resectability ${ }^{b}$} & $\mathrm{R}$ & $23(85)$ & $17(89)$ & 0.671 \\
\hline & $\mathrm{BR}$ & $4(15)$ & $2(11)$ & \\
\hline Alb & & $3.8(2.6-4.6)^{\mathrm{a}}$ & $3.7(2.5-4.6)^{\mathrm{a}}$ & 0.960 \\
\hline $\mathrm{Hb}$ & & $11.5(8.5-14.1)^{\mathrm{a}}$ & $11.9(9.2-16.3)^{\mathrm{a}}$ & 0.292 \\
\hline NLR & & $4.3(1.1-59.0)^{\mathrm{a}}$ & $2.5(0.8-20.4)^{\mathrm{a}}$ & 0.133 \\
\hline \multirow[t]{3}{*}{ Surgical procedure } & $\mathrm{PD}$ & $20(74)$ & $10(53)$ & 0.014 \\
\hline & DP & $4(15)$ & $9(47)$ & \\
\hline & $\mathrm{TP}$ & $3(11)$ & $0(0)$ & \\
\hline Operation time (min) & & $485(327-692)^{\mathrm{a}}$ & $423(220-710)^{\mathrm{a}}$ & 0.044 \\
\hline Blood loss (ml) & & $1438(354-6970)^{\mathrm{a}}$ & $944(152-10564)^{\mathrm{a}}$ & 0.347 \\
\hline Portal vein resection & & $15(56)$ & $5(26)$ & 0.049 \\
\hline Transfusion & & $13(48)$ & $5(26)$ & 0.135 \\
\hline LN metastases & & $12(46)$ & $7(37)$ & 0.532 \\
\hline Resection status (R0) & & $23(88)$ & $19(100)$ & 0.125 \\
\hline Morbidity $^{\mathrm{C}}$ & & $5(19)$ & $2(11)$ & 0.457 \\
\hline POPF ${ }^{\mathrm{d}}$ (Grade B, C) & & $4(15)$ & $5(26)$ & 0.477 \\
\hline $\operatorname{DGE}^{\mathrm{e}}$ (Grade B, C) & & $13(48)$ & $7(41)$ & 0.651 \\
\hline Postoperative Hospital stay (day) & & $33(14-153)^{\mathrm{a}}$ & $28(17-98)^{a}$ & 0.290 \\
\hline Induction of ACT & & $22(81)$ & $12(63)$ & 0.165 \\
\hline Completion of ACT (6 months) & & $10(34)$ & $6(32)$ & 0.702 \\
\hline
\end{tabular}

1a, 1b Comparison of overall survival (OS, Figure 1a) and relapse free survival (RFS, Figure 1b) between the elderly and younger groups. There was no significant difference both in OS and RFS ( $P=0.270$, $\mathrm{P}=0.699$ ). 


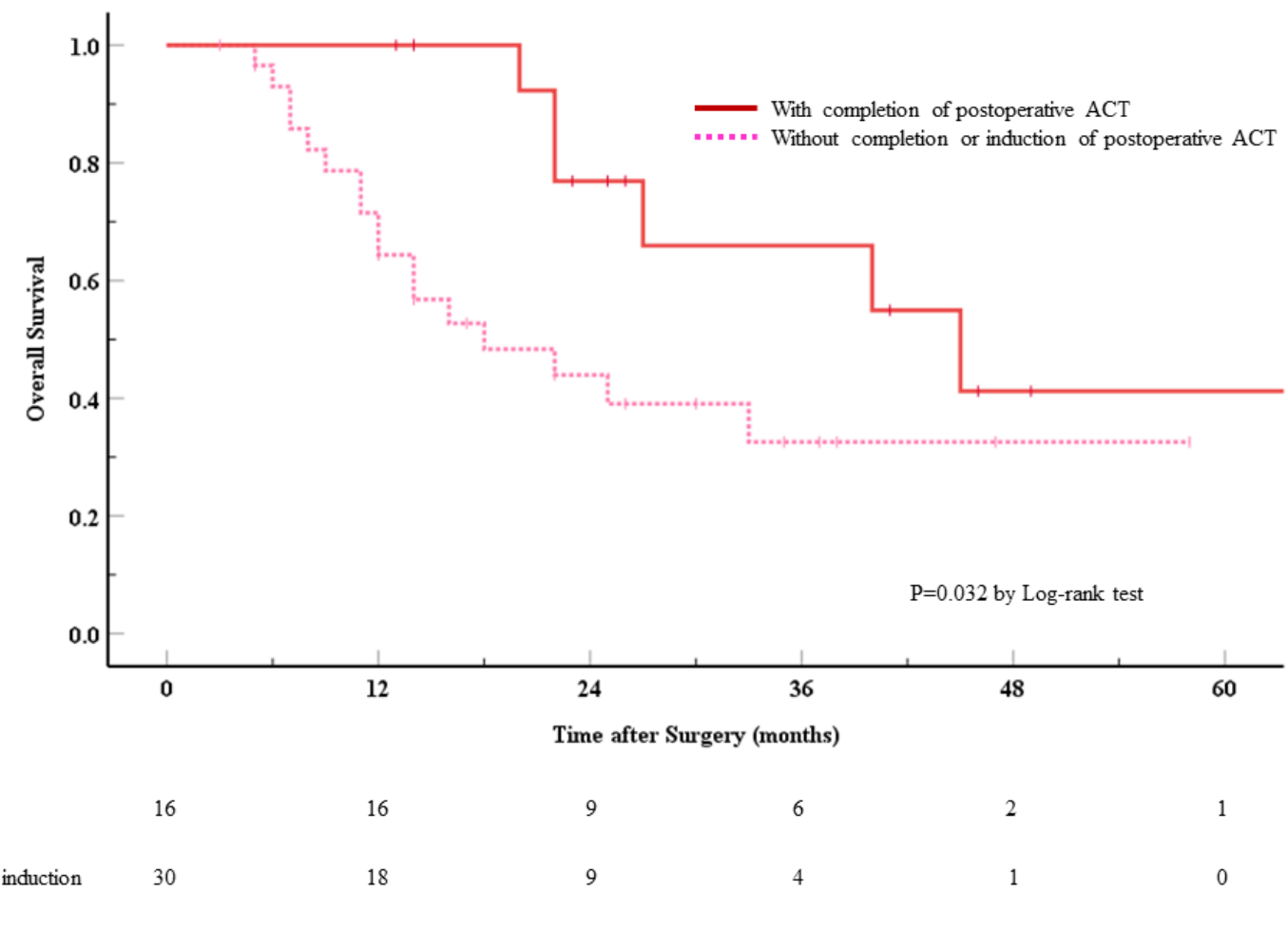

\section{Figure 2}

Comparison of overall survival (OS) in the elderly group between patients with completion of postoperative adjuvant chemotherapy (ACT) and without completion or induction of ACT. The elderly patients with completion of ACT had significantly better OS than those without completion or induction of ACT $(P=0.032)$. 


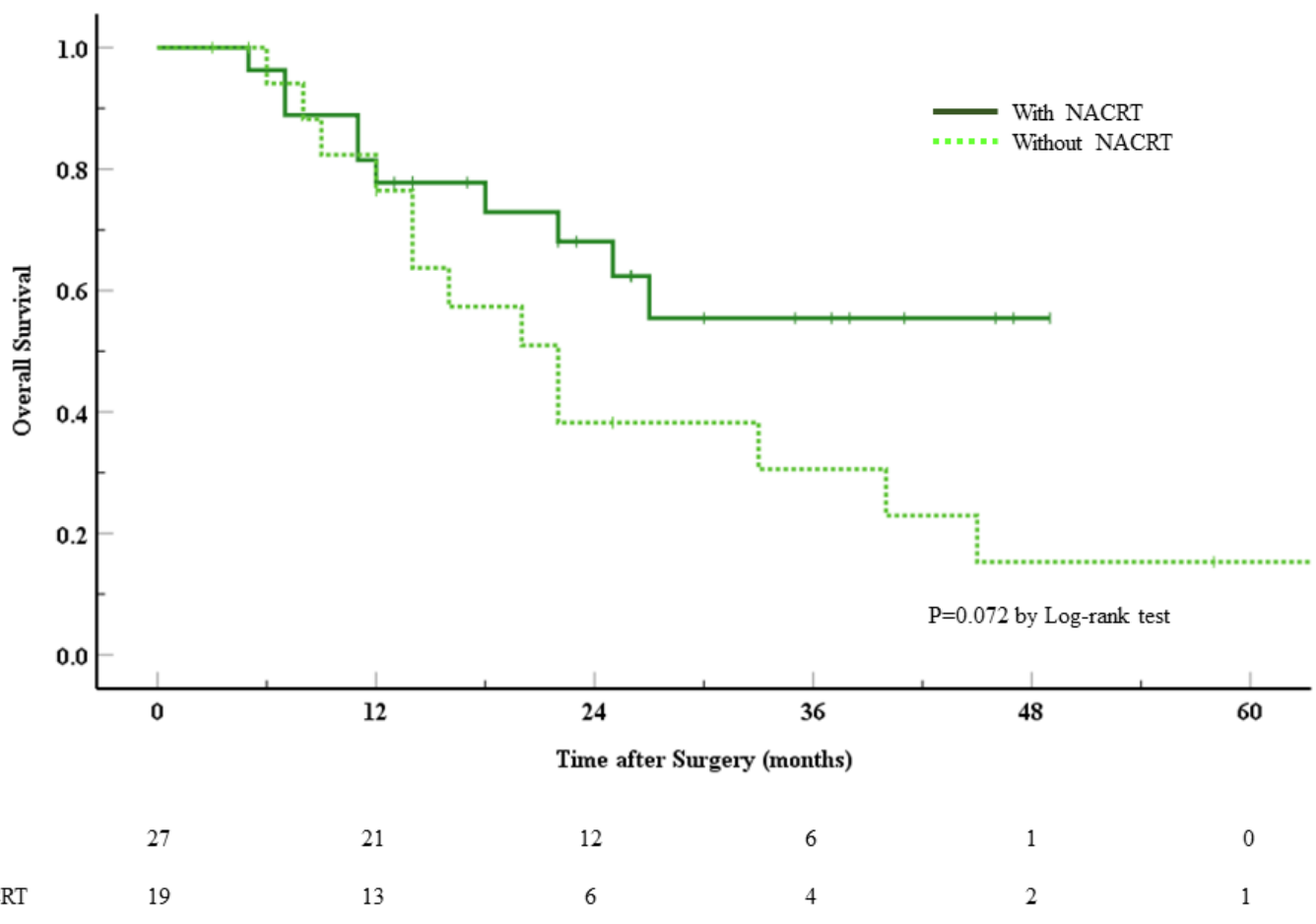

\section{Figure 3}

Comparison of overall survival (OS) in the elderly group between patients with and without preoperative neoadjuvant chemoradiotherapy (NACRT). NACRT did not significantly affect OS in elderly patients, however, there was a trend for improvement of OS $(P=0.072)$. 


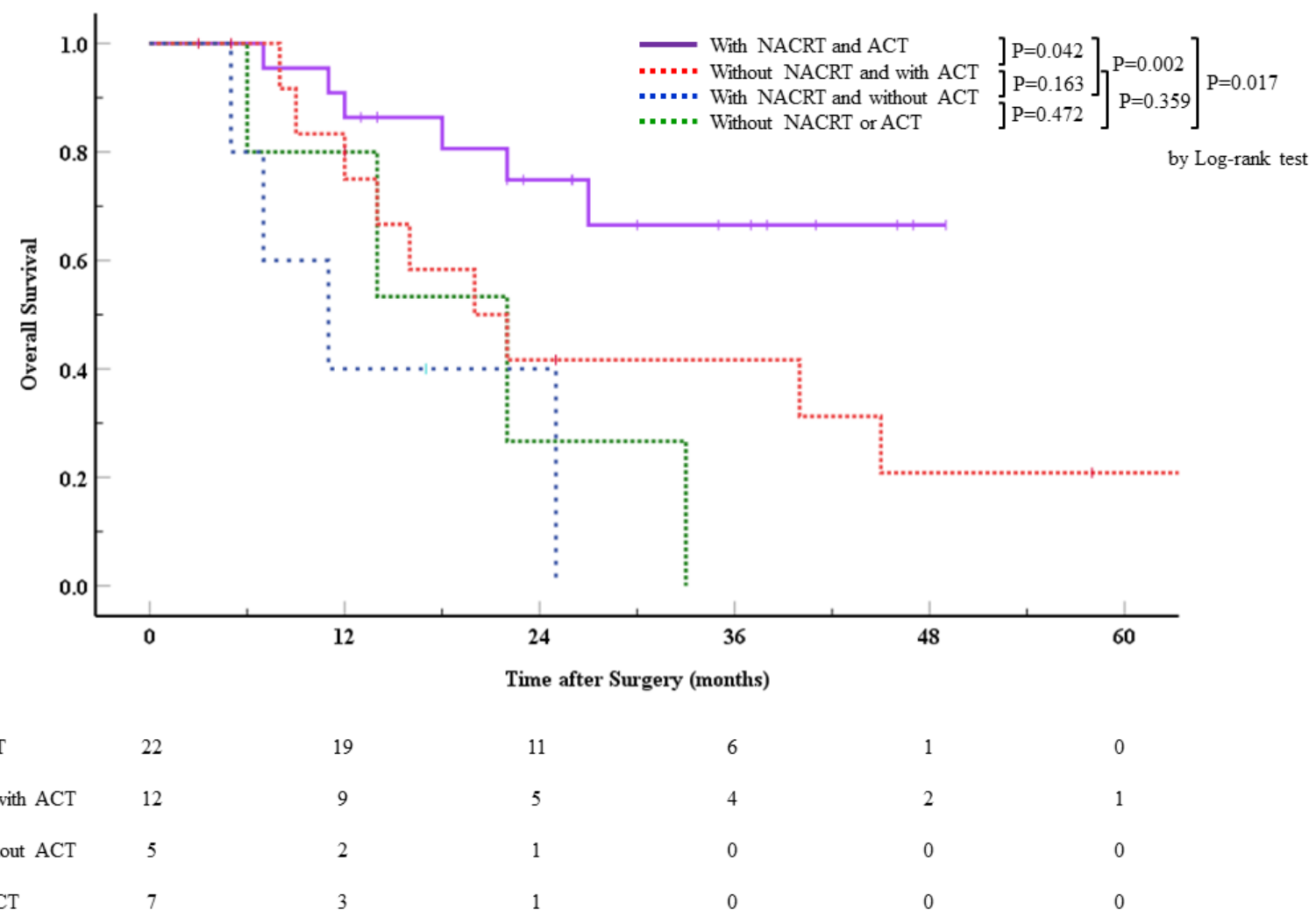

\section{Figure 4}

Overall survival (OS) curves in the elderly group dividing into 4 groups with and without the inductions of preoperative neoadjuvant chemoradiotherapy (NACRT) and postoperative adjuvant chemotherapy (ACT). Only patients who introduced NACRT and ACT had significantly better OS than those who did either of NACRT or ACT or did not do either $(P=0.042, P=0.002$ and $P=0.017)$. 